ISSN: 0213-3563

https://doi.org/10.14201/azafea202123189214

\title{
LA FILOSOFÍA DE LAS INSTITUCIONES DE ARNOLD GE- HLEN. UNA REIVINDICACIÓN
}

\author{
The Philosophy of Institutions of Arnold Geblen. A Vindication
}

Francisco Tadeo BACIERO RuIZ

Universidad de Salamanca

Recibido: $21-05-2020$

Aceptado: 11-12-2020

\section{RESUMEN}

Arnold Gehlen es una de las figuras rectoras de la Antropología filosófica como corriente filosófica característica del siglo XX. En su obra de 1956 Urmensch und Spätkultur [Hombre primitivo y cultura tardía], Gehlen desarrolla una sofisticada "filosofía de las instituciones" en la que discierne su origen y valor antropológico para el ser humano, quien, como ser biológico caracterizado por su fragilidad y "carácter no fijado", necesita de un apoyo exterior para su conducta, apoyo que es proporcionado por las instituciones como marco de un conjunto de acciones reguladas y obligatorias que confiere integración y continuidad a las sociedades humanas. La cultura de la "subjetividad" que invade las sociedades occidentales contemporáneas amenaza la funcionalidad de las instituciones y su carácter protector para la persona.

Palabras clave: Arnold Gehlen; antropología filosófica; acción; instituciones; subjetividad.

\section{ABSTRACT}

Arnold Gehlen is one of the leading figures of philosophical Anthropology as a main philosophical trend of the twentieth century. In his work published in 1956 Urmensch und Spätkulur, Gehlen develops a sophisticated "philosophy of institutions" in which he analyses the origin and anthropological value of institutions for the human being, who, as a biological being characterized for his frailty and "not fixed character", needs of an external 
support for his conduct, support which is provided by institutions as a frame of regulated and compulsory set of actions that confers integration and continuity to human societies. The culture of "subjectivity" that invades contemporary occidental societies threatens the functionality of institutions and its shielding character for the person.

Key words: Arnold Gehlen; philosophical Anthropology; action; institutions; subjectivity.

\section{INTRODUCCIÓN}

De forma algo intempestiva sentenciaba Herbert Schnädelbach en su ensayo sobre la historia de la filosofía alemana entre la muerte de Hegel y el ascenso de Hitler al poder, que la antropología filosófica como corriente filosófica era un producto "típicamente alemán”, un intento más de "rehabilitación de la filosofía como ciencia”, en la línea de otros intentos de recuperación tras la crisis de identidad provocada por el colapso de la filosofía hegeliana. Este intento, paradójicamente, habría contribuido, contra su propia voluntad, a eclipsar el discurso sobre el hombre, disuelto en la filosofía "posantropológica" de Heidegger y de la Escuela de Frankfurt (Schnädelbach, 1991: 265-6, 279-280).

El juicio sumarísimo de Schnädelbach, pronunciado en 1983, no se compadece sin embargo con el resurgir institucional y doxográfico de la antropología filosófica en los últimos treinta años en Alemania ${ }^{1}$. Lo mismo cabría decir de otros países europeos².

Entre los autores fundacionales de la antropología filosófica como "enfoque” o corriente filosófica se encuentra sin duda Arnold Gehlen. Gehlen

1. Se puede apuntar en ese sentido las fundaciones en 1993 de la "Max Scheler Gesellschaft" (cf. www.max-scheler.de), y de la "Helmuth Plessner Gesellschaft" en 1999 ( $c f$. http://helmuth-plessner.de), que han auspiciado una serie de congresos y publicaciones periódicas sobre este enfoque filosófico (sobre la distinción entre la antropología filosófica como enfoque o corriente filosófica -"philosophischer Ansatz"-, y la antropología filosófica como disciplina académica - "philosophische Disziplin"-, cf. Fischer, 2009 , passim). Entre las grandes monografías alemanas sobre la materia es imprescindible señalar, además de Fischer (2009), Fischer (2016).

2. Cf. por ejemplo en Italia los estudios de Pansera $(1991,2001)$, así como la traducción de Moral und Hypermoral en Gehlen (2001) (estas y otras referencias en Lombo, 2008). En Francia se acaban de presentar sendas traducciones de las dos obras clásicas de Plessner y Gehlen (Plessner, 2017, y Gehlen, 2021). 
tuvo la virtud de mantener vivo el paradigma de la antropología filosófica en la Alemania de posguerra hasta los años setenta del siglo pasado, cuando las otras dos figuras fundacionales del enfoque (Scheler y Plessner) habían caído en el olvido, eclipsadas tanto por el empuje arrollador de la filosofía de la existencia de Heidegger, como por la muerte de Scheler en 1928 y el exilio forzado de Plessner en 1933 por su condición de "medio judío", de acuerdo con la jerga nazi (Marquard, 1965, 2007). A raíz de la publicación en 1956 de Urmensch und Spätkultur y de Moral und Hypermoral en 1969, así como de las vivas discusiones que suscitó su crítica conservadora de la cultura entre miembros eminentes de la Escuela de Frankfurt como Habermas y Adorno, Gehlen consiguió además una amplia repercusión entre el público culto de la época.

La relevancia de la antropología de Gehlen para la sociología (y en concreto de su teoría de las instituciones), fue sugerida por Peter Berger y $\mathrm{H}$. Kellner en un artículo de 1965 en el que, tras una sucinta exposición de su teoría antropológica, apuntaban las líneas de la presencia de Gehlen en la sociología alemana de posguerra, tanto en su discípulo y antiguo asistente Helmuth Schelsky, como en Dieter Claessens y otros; al tiempo que lamentaban el desconocimiento de su obra entre los sociólogos anglosajones (Berger y Kellner, 1965) ${ }^{3}$. Joachim Fischer ha esbozado en un denso ensayo las líneas de la larga historia de la eficacia de la antropología de Gehlen en la sociología alemana, que se extiende no sólo a Schelsky, sino también a Niklas Luhmann, doctorando de Schelsky, al mencionado Dieter Claessens y al propio Habermas (Fischer, 2006) . $^{4}$

En España la antropología filosófica de Gehlen se conoció muy tardíamente y ha alcanzado relativamente poca repercusión. Con la excepción de la publicación de su manual de sociología de 1955 editado en colaboración con Schelsky (Gehlen, Schelsky y Jantke, 1962), su obra clásica sobre el hombre

3. Un año más tarde, en su obra clásica sobre sociología del conocimiento, Berger y Luckmann identificarán las doctrinas de Plessner y Gehlen como la base antropológica de su sociología simbólica: "Nuestros presupuestos antropológicos tienen gran influencia de Marx [...] y de las inferencias antropológicas extraídas de la biología humana por Helmuth Plessner y Arnold Gehlen” (Berger y Luckmann, 1986: 32).

4. Cf. además Raulet, 2009: 91-105. Alvarez Munárriz (1996) ha esbozado las líneas de la influencia de Gehlen, además de en la sociología de Luhmann, en la socióloga marxista Agnes Heller, que se inspira en su bioantropología para su crítica de las interpretaciones etológicas del hombre. 
de 1940, no se tradujo hasta 1980 (Gehlen, 1980)5. Tras ella se sucedieron las publicaciones de algunos ensayos sobre antropología filosófica y crítica cultural y estética (Gehlen, 1988, 1993, 1994). Los estudios sobre su obra tampoco son abundantes ${ }^{6}$. La teoría de las instituciones en concreto apenas si ha sido objeto de un tratamiento pormenorizado, con excepción de los dos estudios de Sánchez Capdequî́. El presente trabajo pretende una presentación sistemática y contextualizada de la doctrina gehleana sobre las instituciones que completa y amplía en diversos aspectos los estudios previos de Sánchez Capdequí.

\section{LA ANTROPOLOGÍA DE LA ACCIÓN DE GEHLEN COMO FUNDAMENTO DE SU FILOSOFÍA DE LAS INSTITUCIONES}

Se podría decir que la teoría de las instituciones de Gehlen es el complemento sociológico de su doctrina sobre el hombre, calificada con razón como una "antropología de la acción", es decir, de la inevitable transformación "activa” del entorno a la que el hombre se ve abocado en su interacción con el mundo como compensación inevitable de sus "carencias biológicas". Se trata de una categoría interpretativa básica que Gehlen había anticipado ya en un ensayo de 1935 como núcleo de una posible antropología filosófica concebida como "filosofía primera" .

Como ser biológicamente carencial [Mängelwesen], desde el punto de vista tanto de su inespecialización morfológica como de su carencia de ver-

5. Las citas en castellano de esta obra clásica de Gehlen estarán tomadas en lo sucesivo de esta edición publicada en Salamanca en 1980.

6. Sin ánimo de ser exhaustivos, podemos mencionar en orden cronológico los de González-Jara, 1970; Rodríguez Molinero, 1989; Álvarez Munárriz, 1996; Sánchez Capdequí, 1997a y 1997b; Lombo, 2008; Rosales Rodríguez, 1999, y Miranda Rebeco, 2012 y 2012.

7. Especialmente valioso nos parece Sánchez Capdequí (1997b), que proporciona además un repertorio bibliográfico sociológico sobre el tema del que nos hemos podido beneficiar para el presente trabajo (muy especialmente del estudio de Lourau, 1988, así como de la obra clásica de Mead, 1967).

8. Una buena exposición crítica de conjunto de la antropología de Gehlen puede verse en Böhler, 1981; también es de utilidad la propia autoexposición de Gehlen, 1957, y Rodríguez Molinero, 1989.

9. "En las siguientes reflexiones examinamos una doctrina del actuar humano ["eine Lehre vom menschlichen Handeln”] como la parte nuclear de una antropología filosófica, y a esta misma como prima philosophia o ciencia filosófica fundamental” (Gehlen, 1980b: 311). 
daderos instintos, el hombre es siempre el "animal no fijado" (Nietzsche) $)^{10}$. El hombre se ve obligado a compensar sus deficiencias mediante sus propias fuerzas a través de una conducta planificada, es un ser "destinado por naturaleza a la acción” 11 .

El animal no "actúa” propiamente desde sí o por sí mismo, en la medida en que su dotación instintiva determina por adelantado tanto la parte de su "mundo circundante" que es capaz de percibir en función de sus necesidades biológicas, como las reacciones motóricas automáticas acordes con sus percepciones. El animal no es consciente del sentido e intencionalidad de sus acciones, que se desencadenan "por la espalda de su conciencia” (Gehlen, 1966: 403).

El hombre, en cambio, tiene que adquirir por sí mismo todas las capacidades que le permitirán enfrentarse al mundo; en primer lugar, sus complejas capacidades motóricas, que debe adquirir por su propio esfuerzo, incluso la marcha erguida, que, lejos de ser una dotación "natural”, necesita ser aprendida (Gehlen, 1966: 180ss.).

Especialmente debe el hombre adquirir el control de su vida pulsional. Por su carencia de instintos en sentido estricto, padece de una "sobrecarga pulsional" [Triebüberschuss] ${ }^{12}$, en el sentido de que sus pulsiones, a diferencia de lo que ocurre en los animales, ni están pautadas cíclicamente ni se transforman automáticamente en acciones consumatorias ligadas a la satisfacción de su fin biológico específico (acciones que suprimen la pulsión una vez ejecutadas). Al contrario, por falta de regulación instintiva, las pulsiones biológicas pueden desvincularse en el hombre de su fin biológico natural y conducirle a una "proliferación lujuriosa" [luxurieren], neologismo que Gehlen acuña

10. La noción de "ser carencial”, como el propio Gehlen reconoce en la introducción a El hombre, es original de Herder. Al final del apartado primero de la primera parte de su Tratado sobre el origen del lenguaje de 1772, Herder caracteriza en efecto al ser humano en comparación con el reino animal, como un ser afectado por "lagunas y carencias" [Lücken und Mängel], que sólo es capaz de compensar gracias a su competencia lingüístico-racional (Herder, 1964: 15-19).

11. El hombre es "un ser práxico" (Gehlen, 1980a: 36), "el proyecto natural de un ser actuante" ["Naturentwurf eines handelnden Wesens"] (Gehlen, 1966: 2; en adelante citaremos el texto original alemán por esta edición); y en consecuencia: "La autodisciplina, la educación, el adiestramiento en el sentido de adquirir forma o mantenerse en ella; todo ello pertenece a las condiciones de existencia de un ser no terminado" (Gehlen, 1980a: 35-36).

12. El concepto de “Triebüberschuss” sería original de Seidel, 1927: 216 (cf. Fischer, 2009: 47, nota 75). 
para la ocasión (Gehlen, 1966: 351); una proliferación que sin diques reguladores amenaza circunstancialmente con destruirlo. Si las pulsiones biológicas se traducen en el animal de forma automática y predeterminada en la correspondiente conducta instintiva, el hombre "abandonado por los instintos" no sabe de entrada qué hacer con ellas, y en este caso su aprendizaje tendrá por objeto la regulación o capacidad de "disciplinar" su vida pulsional. Por ello mismo el hombre, además de un ser destinado al aprendizaje [Lernwesen], es un ser capaz de y necesitado de disciplina o "amaestramiento" [Zuchtwesen] (Gehlen, 1966: 32, 356ss).

Las deficiencias que afectan al ser humano desde un punto de vista biológico son, como hemos dicho, compensadas por sus propias acciones y por los productos duraderos de sus acciones, el conjunto de los cuales constituyen lo que de un modo genérico se denomina "cultura", que se convierte en su "segunda naturaleza", auténtico "mundo circundante" y nicho "natural" del hombre ${ }^{13}$.

Las instituciones, como veremos a continuación, conforman el núcleo esencial o la "gramática" del mundo cultural, proporcionando estabilidad y continuidad a todas las creaciones humanas, facilitando su reproducción y transmisión indefinida, al tiempo que fungen como apoyo externo regulador de la difícil vida pulsional de un ser aquejado por una peligrosa plasticidad, a modo de "refuerzos exteriores" que "hacen fiable la parte interna de la moral” (Gehlen, [1957] 1993: 39-40) ${ }^{14}$.

\section{LA FILOSOFÍA DE LAS INSTITUCIONES COMO TAL}

En el lenguaje cotidiano la palabra "institución" evoca nociones como “permanencia”, “estabilidad”, carácter colectivo y "regulación”, propiedades que en virtud de la fuerza institucional, se superponen a una realidad circundante carente de ellas ${ }^{15}$.

13. "Como consecuencia de su primitivismo orgánico y su carencia de medios, el hombre es incapaz de vivir en cualquier esfera de la naturaleza realmente natural y original. [...] La esencia de la naturaleza transformada por él en algo útil para la vida se llama cultura y el mundo cultural es el mundo humano” (Gehlen, 1980a: 42-43).

14. Habermas, buen conocedor de la antropología filosófica desde su época de estudiante en Bonn, atribuye aproximadamente la misma función al derecho: "El derecho puede compensar las debilidades de una moral exigente que, sin embargo, cuando se consideran las consecuencias empíricas, sólo proporciona resultados cognitivamente indeterminados y motivacionalmente inciertos" (Habermas, 1999: 251).

15. Estas nociones coinciden en lo esencial con el sentido etimológico del término: 
Cualquier "institución” prescribe efectivamente modos de actuar obligatorios a determinados tipos de conducta: en el siglo II los doce libros Institutionis oratoriae de Quintiliano prescribían normas al discurso forense o político en la Roma imperial; las Institutiones grammaticae de Prisciano la sintaxis normativa de la lengua latina en el siglo VI, las Institutiones del derecho romano recopiladas por Gayo en el siglo II prescribían conductas de obligado cumplimiento (u obligada abstención) jurídicamente sancionadas, como lo harán cuatro siglos más tarde las Institutiones Iustianiani recopiladas por el emperador bizantino.

El campo semántico al que remitía el sustantivo "institución”, que en un principio parece haberse restringido al terreno de la ordenación o fundación de determinadas ramas del saber ${ }^{16}$, se trasladará con el tiempo al terreno social, pasando a designar los mecanismos ordenadores o reguladores de determinadas interacciones humanas. Al menos desde finales del siglo XVIII encontramos en Francia un uso genérico del término “institución” aplicado a fenómenos sociales políticos, civiles o religiosos dotados de cierta estabilidad y que recoge en lo esencial el semantema de la palabra que ha permanecido invariable prácticamente hasta hoy ${ }^{17}$. En el manifiesto antiliberal de 1814, Essai sur les principes générateurs des constitutions politiques et des autres institutions humaines, Joseph de Maistre usará el término con idéntico significado y formando parte de los mismos sintagmas de los que podemos encontrarlo formando parte todavía hoy ${ }^{18}$.

el sustantivo latino institutio-onis significa "disposición", "plan", "formación", "instrucción", "institución". El verbo de la tercera conjugación instituo -ere -tuti-tutum, por su parte: "poner" (las plantas del pie sobre el suelo), "preparar", "disponer", "formar" (las tropas en orden de batalla), "establecer", "educar" (Vox, 1990: 253).

16. Todavía Leonhard Euler titulará sus trabajos de constitución o fundamentación de algunas ramas de las matemáticas: Institutiones Calculi Differentialis (1765), o Institutiones Calculi Integralis (1768-1770).

17. En ese sentido lo emplea ya Diderot en 1771 en su Suplemento al viaje de Bougainville cuando se refiere al "yugo" de las "instituciones políticas, civiles y religiosas" diseñadas por "un puñado de bribones": "J'en appelle à toutes les institutions politiques, civiles et religieuses, examinez-les profondément; et je me trompe fort, ou vous verrez l'espèce humaine pliée de siècle en siècle au joug qu'une poignée de fripons se promettait de lui imposer" (Diderot, 1771: 48-49, esta referencia y buena parte de las siguientes están tomadas del detenido estudio de conjunto de Lourau, 1988: 23-144). Saint-Just en sus Fragments sur les institutions républicains, de 1794, afirmará que "es necesario sustituir, mediante las instituciones la influencia personal [del monarca] por la fuerza y la justicia inflexible de las leyes" (en Lourau, 1988: 36).

18. De Maistre define el cristianismo como "la plus grande des institutions imagina- 
Como término casi técnico en la jerga de las ciencias humanas (y de la sociología en particular), podemos encontrar "institución” ya en el Curso de filosofía positiva de Comte de 1830, que se refiere en diversas ocasiones a la "institución" de la familia o del capital (Roggero, 1986: 925). Hipólito Taine, en carta a Guizot, convierte a las instituciones (en el mismo sentido en el que lo hará Gehlen), en garantes y condición de posibilidad de una vida civilizada ${ }^{19}$.

Aproximadamente un siglo después se iniciará el estudio de las instituciones en la sociología teórica y la antropología cultural. Los precursores parecen haber sido también franceses, y en concreto los juristas Hauriou y Renard, quienes subrayarán (en la línea en que lo habían hecho los pensadores tradicionalistas), el carácter fundacional y estabilizador que tienen las instituciones para las sociedades ${ }^{20}$.

En Estados Unidos, y aparentemente de forma independiente de sus inmediatos predecesores franceses, Mead y Malinowski inician el estudio de las instituciones desde el punto de vista de la psicología social y la antropología cultural. En su estudio clásico publicado en 1934, Mead las define como un sistema de acción organizado que suscita expectativas recíprocas en el comportamiento de todos los miembros de un grupo ${ }^{21}$. Malinowski, que

bles" (De Maistre, 1814: 20), y en diversos lugares se refiere a las: "institutions établies" (De Maistre, 1814: 61), "institutions politiques" (De Maistre, 1814: 67), "institutions nationales" (De Maistre, 1814: 94), "institutions sociales" (De Maistre, 1814: 100), "institutions civiles et religieuses" (De Maistre, 1814: 100).

19. "La historia muestra que los gobiernos, las religiones e iglesias, todas las grandes instituciones son los únicos medios a través de los cuales el hombre animal y salvaje (l'homme animal et sauvage) alcanza su pequeña porción de razón y justicia" (en Gehlen, 1973: 100, que lo toma de De Jouvenel, 1955: 295).

20. "Tanto en el derecho como en la historia, las instituciones representan la categoría de la duración, de la continuidad y de lo real; la operación de fundarlas constituye el fundamento jurídico de la sociedad y del Estado" (Hauriou, 1925, en Lourau, 1988: 53); "son las instituciones las que hacen las reglas del derecho, y no las reglas jurídicas las que hacen las instituciones" (en Lourau, 1988: 61); "Permanencia en la sucesión del tiempo, unidad de ser en la diversidad de los órganos: eso es la institución" (Renard, 1930, en Lourau, 1988: 64).

21. "The institutions of society are organized forms of group or social activity forms so organized that the individual members of society can act adequately and socially by taking the attitudes of others toward these activities" (Mead, 1967: 261-2). En un estudio temático ocho años posterior, se introduce la perspectiva funcionalista: la institución sería una "estructura social y el mecanismo con el que la sociedad humana organiza, dirige y desarrolla las actividades multiformes que se requieren para satisfacer 
promoverá la perspectiva funcionalista, las definirá de forma muy parecida como "una organización de seres humanos con un fin predado al que se someten y que es reconocido por la sociedad" (Malinowski, 1949: 85) 22. Talcott Parsons, de forma algo más imprecisa, hablará de ellas en El sistema social (1951) como subsistemas del sistema o estructura social total, tendentes a estabilizar ciertos roles convirtiéndolos en obligatorios para determinados grupos sociales, cuyo contraconcepto límite sería el de anomia:, un comportamiento no atenido a norma, expectativa o restricción alguna ${ }^{23}$.

En Alemania Gehlen dará a conocer su antropología de las instituciones en Urmensch und Spätkultur [Hombre primitivo y cultura tardía], de 1956, cuyas tesis esenciales retomará en 1969 con fines polémicos en Moral und Hypermoral [Moral e hipermoral], al hilo de la exposición de su ética antropológica de trasfondo biológico.

Ya en el capítulo 44 de la edición de 1950 de El hombre (titulado "Algunos problemas del espíritu”, que reelaboraba por su parte el último capítulo de la edición original de 1940, "Oberste Führungsysteme", "Sistemas superiores de dirección”), había propuesto Gehlen un esbozo de su teoría de las instituciones ${ }^{24}$.

En dicho capítulo proponía el análisis de los aspectos sociales de su doctrina antropológica, al constatar cómo fenómenos tradicionalmente considerados pertenecientes a las "ciencias del espíritu" ("religión, arte, derecho, técnica”), estudiados tradicionalmente con los métodos de las ciencias “comprensivas", dependían en su legalidad de determinados mecanismos sociales. Así lo habría demostrado Weber para el caso de las religiones, razón por la que su estudio requeriría de la ayuda de la sociología. Por esa razón el estudio científico de los "mundos" del "espíritu objetivo" en su "concreción

las necesidades humanas” (Barnes, 1942, en Roggero, 1988: 926).

22. En realidad "la ciencia real del comportamiento humano tiene que comenzar con la organización” (Malinowski, 1949: 83).

23. "Una institución es un complejo de integraciones de rol institucionalizadas que tiene significación estructural en el sistema social" (Parsons, 1989: 46, cursivas en el original); "La antítesis polar de la completa institucionalización es la anomia: la ausencia de complementariedad estructurada del proceso de interacción o, lo que es igual, el quebrantamiento completo del orden normativo" (Parsons, 1989: p. 46).

24. La historia editorial de El hombre puede leerse en Rehberg (1983: 751-5); la edición considerada definitiva es la sexta de 1962, que contiene ligeros retoques respecto a la cuarta de 1950. 
histórica” necesitaría ser completado por el estudio de su "dimensión sociológica" (Gehlen, 1980a: 449) 25

La elaboración sistemática de una teoría de las instituciones quedará reservada con todo a Urmensch und Spätkultur ${ }^{26}$, como hemos dicho. La teoría elaborada en esta obra será completada al año siguiente con las observaciones sobre la sobrecarga de "subjetividad" a que se ve expuesto el hombre en la cultura occidental, recogidas en el ensayo Die Seele im technischen Zeitalter [El alma en la edad técnica] (Gehlen, 2004).

Analizaremos a continuación con algún detenimiento las que Habermas considerará "observaciones ingeniosas y agudísimas que van esparciéndose incidentalmente", junto con "consideraciones profundas sobre la esencia de la ascesis" (Habermas, 2019: 109, 111) a lo largo de USK; una investigación que "busca las categorías antropológicas de las instituciones" (Gehlen, 1956: 21) en el marco de una "filosofía empírica" (Gehlen, 1956: 3). Con ellas Gehlen pretende elaborar una "filosofía de las instituciones", que incluya la pregunta por su "origen y formas primarias", formas que intentará rastrear en las culturas primitivas (Gehlen, 1956: 9) ${ }^{27}$.

Gehlen aspira a lograr un doble objetivo con su investigación sobre las instituciones: proponer, de un parte, una teoría sobre su posible génesis ob-

25. Gehlen traía a colación en este contexto sendos pasajes de Ruth Benedict y Malinowski. Parece que Gehlen entró en contacto con la antropología cultural norteamericana, muy presente en su obra desde entonces, gracias al acceso que se le facilitó en 1947, con su discípulo Schelsky, a la American Library de Karlsruhe (cf. Wiki deutsch, 2020, que remite a Rehgberg, 2013: 18). Esa es la razón de que en la edición de 1950 de El hombre se encuentren diversas referencias a la teoría del "otro generalizado" de G. H. Mead, que darán a conocer al psicólogo social norteamericano por vez primera en Alemania (Fischer, 2009: 229), hecho nada desdeñable si tenemos en cuenta la importancia capital que Mead desempeñará en la sociología de Habermas, estudiante por entonces de veintiún años.

26. El libro conocerá otras dos ediciones ligeramente revisadas en vida del autor, en 1964 y 1975. Gehlen había concebido USK como la continuación lógica de El hombre, hasta el punto de que había pensado titularla "Der Mensch. Bd. II", pero renunció al título por consejo de su editor (Rehberg, 1993: 914).

27. En ningún momento llega Gehlen a identificar la lista de entes colectivos que quedarían subsumidos en la categoría "institución”, si bien, a partir de los ejemplos que aduce de forma asistemática, se incluirían entre ellos al menos los siguientes: el matrimonio y la familia, el trabajo socialmente regulado en cualquier época (campo, industria, funcionariado), los sistemas de educación reglada, las organización políticas, el estado, el derecho (la institución que "sella" la existencia de otras instituciones), y, en última instancia, como veremos, también el lenguaje. 
jetiva (en el sentido de independiente de cualquier plan o acción deliberada) $y$, de otra, dar cuenta de su funcionalidad (estricta necesidad en realidad) para la vida humana, para concluir con una decidida apología de las instituciones frente a la tendencia al "subjetivismo" que amenaza su existencia en las sociedades occidentales desarrolladas.

Para Gehlen, como para Marx, el hombre es un ser de necesidades para cuya satisfacción necesita transformar el mundo. Como medio inmediato de dicha transformación el hombre se sirve de entrada de herramientas.

La construcción de una herramienta supone ya una capacidad de abstracción, en la medida en que entre el deseo biológico de comer y su consumación en el futuro gracias a la presa cazada por el uso de un cuchillo de piedra fabricado con el fin de matar animales, se ha producido un proceso de abstracción de la situación o necesidad biológica inmediata en el que aparecen "intereses o necesidades" secundarias (fabricar el cuchillo) respecto a las primarias (comer) ${ }^{28}$.

Gracias a la fabricación de herramientas, convertida progresivamente en actividad habitual o costumbre que tiende a perpetuarse en el tiempo como actividad rutinaria (y la posterior división del trabajo acontecida en el neolítico, que obliga a la especialización en trabajos complementarios para la supervivencia del grupo), aparece un nuevo fenómeno antropológico, a saber: la actividad fabricadora puede prescindir o "descargarse" de su fin primario (la satisfacción de la necesidad biológica a la que sirve como medio), para convertirse en una actividad buscada en virtud de lo que Gehlen llama un "comportamiento experimentante" [experimentierendes Verhalten]. Que este se ejecuta por mor del placer que el sujeto halla en el propio comportamiento, independientemente de la finalidad biológica a la que originalmente sirve. Se produce así el fenómeno de la "separación del motivo [de la acción] de la finalidad [biológica]", gracias al cual la acción misma se convierte en “fin por sí mismo" (Gehlen, 1956: 31) ${ }^{29}$.

Gracias a la mentada separación, el comportamiento técnico "descargado" del fin biológico permite un enriquecimiento de motivos y de conductas perfeccionadoras, que quedan "liberadas" para metas más complejas y sofisticadas. En ello radica precisamente la posibilidad del perfeccionamiento de

28. En ese sentido la construcción de herramientas es ya un comportamiento "inteligente” y las herramientas paleolíticas serían “conceptos pétreos”, por así decir ("steinerne Begriffe”) (Gehlen, 1956: 11, 13).

29. Cf. todo el cap. 7 “Actuar como fin en sí mismo” (Gehlen, 1956: 29 ss.). 
la cultura en cualquier terreno. En efecto, a partir de cierto nivel de desarrollo de las habilidades técnicas, llega el momento en que se diría que "se come" no para "alimentarse", sino para estar en condiciones de llevar a cabo el trabajo necesario para el buen funcionamiento del trabajo o de la institución dentro de la que se trabaja. El objeto construido tiene en sí un "poder desencadenante", convirtiéndose en una "especie de actualizador crónico" (Gehlen, 1956: 26) de la conducta que lo fabricó, lo que contribuye a la perpetuación de la conducta el tiempo ${ }^{30}$.

Las actividades productivas distribuidas entre los miembros de la sociedad (también las actividades rituales del culto), se vuelven con el tiempo cada vez más estereotipadas, y en la medida en que adquieren paulatinamente cierto cariz imperativo, se impregnan paso a paso de las propiedades de estabilidad y obligatoriedad que caracterizan las acciones reguladas por instituciones. El mecanismo de la "separación del motivo de la finalidad", por su parte, permite que el trabajo regulado institucionalmente y la propia institución se conviertan ellos mismos en motivos impulsores y perfeccionadores de la conducta. Por ello mismo, las instituciones habrían surgido de un modo natural y no premeditado a partir de conductas cotidianas que con el tiempo se habrían convertido en costumbres estables y regladas, lo que facilitaría su reiteración. No se crearon por tanto como resultado de una acción premeditada o del acuerdo deliberado entre quienes las integran, por mucho que a posteriori pueda explicarse o legitimarse teóricamente su génesis y utilidad, de forma parecida a como la filosofía política surgió mucho tiempo después de que existiesen instituciones políticas vigentes y activas.

El conjunto de momentos o rasgos que definiría las instituciones sería por tanto el siguiente:

la independización y habitualización de grupos de motivos y procesos de acción; su virtual disociación de fines por medio de nuevos fine emergentes; el estado de satisfacción estacionario de necesidades primarias que desaparecen del campo motivacional, la trasposición de los momentos impulsivos en el objeto del comportamiento y la función de apelación o exigencia proveniente de él. (Gehlen, 1956: 39) $)^{31}$

30. Lo mismo sucede con cualquier trabajo especializado, por eso el "funcionario jubilado", por ejemplo, añora su despacho de trabajo, sus actas y las rutinas que acompañaban toda su jornada laboral.

31. El juego es un modelo de actividad institucionalizada, en cuanto supone "un sistema de acciones relacionadas entre sí orientadas a una tarea determinada" (Gehlen, 1956: 42), que dirigido por un sistema de reglas compartidas facilita la realización de una tarea 
Hasta aquí la teoría de Gehlen sobre el probable origen de las instituciones en la prehistoria de la humanidad, que da cuenta de lo que con cierta libertad podríamos llamar sus causas materiales (herramientas, materiales), eficientes (acciones habituales), y formales (reglas de conducta obligatorias).

Cabe preguntarse también por la causa final de las instituciones, su télos inconsciente sin el que su surgimiento y pervivencia no serían explicables.

Para Gehlen las instituciones existen porque sirven a la vida humana en general. Su utilidad o valor antropológico reside en el hecho de que satisfacen las necesidades biológicas humanas de modo duradero, proporcionando al mismo tiempo seguridad y estabilidad a la conducta. Las instituciones proporcionan una "infraestructura de estabilidad y continuidad" a la vida humana (Gehlen, 1956: 27).

Las instituciones tienden a estabilizar la conducta humana de diversos modos. Cumplen en primer lugar la función de lograr una "seguridad de la existencia" a través de lo que Gehlen denomina "satisfacción de trasfondo" ${ }^{2}$, una "categoría antropológica" novedosa que Gehlen pretende haber descubierto $^{33}$. En efecto, el hombre, como cualquier ser vivo, necesita satisfacer necesidades biológicas, pero a diferencia de cualquier otro ser vivo, lo hace de un modo pre-visor o proyectivo, en la medida en que su desvinculación o independencia del bic et nunc espacio-temporal, que hace posible su "apertura al mundo", le permite proyectar su vida en el futuro y angustiarse por adelantado por sus necesidades futuras ${ }^{34}$. Lograr por tanto una "satisfacción de trasfondo" de esas necesidades (la certeza de su satisfacción virtual a largo plazo), le proporciona la seguridad para "descargarse" de la preocupación permanente por ellas, liberando su atención y energías para fines y propósitos a largo plazo, fines y propósitos que no podría plantearse sin la seguridad vital garantizada por la satisfacción de trasfondo.

Todos los descubrimientos que a lo largo de la historia de la humanidad han contribuido a lograr una satisfacción de trasfondo de cualquier tipo han

delimitando "una red de posibilidades" de acción (ejemplo que Gehlen toma en préstamo expresamente de Mind, Self and Society de Mead).

32. "Hintergrundserfüllung" (cf. el cap. 12, "Satisfacción de trasfondo", Gehlen, 1956: 56 ss.).

33. Por "categoría" antropológica entiende Gehlen los "conceptos fundamentales" que dan razón de "las propiedades esenciales irreductibles del hombre" (Gehlen, 1956: 8).

34. "El hambre futura ya le da hambre" (Hobbes, De homine, X, 3, en Gehlen, 1956: 58). 
desempeñado por ello un papel clave en el proceso de humanización: el descubrimiento de la conservación del fuego en primer lugar como instrumento de defensa y protección siempre disponible, o el vestido y la vivienda, que protegen de las inclemencias meteorológicas de forma indefinida. El descubrimiento de la agricultura y la ganadería, sobre todo, permitió "descargar" al hombre neolítico de la permanente preocupación actual por el alimento, facilitando la progresiva especialización del trabajo, y con ella, una satisfacción de trasfondo cada vez más amplia.

La seguridad de trasfondo satisfaría sin embargo no sólo necesidades de tipo material, sino también otras de orden psicológico, como la necesidad de conexión o relación [Zusammenhang], de "proximidad duradera" (de "apego", diríamos, expresado en términos de la psicología evolutiva actual) que todos los hombres experimentan desde la niñez, y que se ve satisfecha de entrada en principio por unas relaciones maternofiliales seguras. El anhelo de conexión o afiliación sería satisfecho en el hombre adulto gracias a su integración en entornos institucionales diversos, en la medida en que las instituciones crean marcos de interacción permanentes y regulados entre personas.

La satisfacción de trasfondo requiere por otra parte la continuidad previsible de las conductas que la hacen posible (la continuidad del trabajo agrícola, por ejemplo), una continuidad que es facilitada por las instituciones en cuanto estabilizadoras de las acciones, prescribiendo determinados comportamientos y prohibiendo otros, en particular las instituciones fundamentales de la familia, el matrimonio y la división del trabajo ${ }^{35}$.

La función estabilizadora de las instituciones no se agota empero en el terreno de las interacciones sociales, sino que se extiende también al ámbito de la conducta individual no directamente orientada a la interacción social, y en concreto a la vida pulsional y moral.

Para Gehlen el hombre es, como acabamos de ver, un viviente aquejado por un exceso de pulsiones biológicas no reguladas que corren el riesgo de degenerar en una "proliferación lujuriosa" ${ }^{36}$. La conducta humana se ve privada en relación con las pulsiones biológicas del papel protector que el instinto desempeña para el animal, papel que consiste en orientarlas median-

35. "Son las instituciones estables fundamentales como el matrimonio y la familia, la división del trabajo, la provisión que se reproduce a sí misma continuamente (trabajo del campo, etc.), las que garantizan la seguridad de la satisfacción de trasfondo para el individuo" (Gehlen, 1956: 60).

36. Vid. supra. 
te conductas estereotipadas hacia una acción consumatoria con un sentido biológico supervivencial que hace desaparecer la pulsión. En ese sentido el animal no puede caer en una "proliferación lujuriosa", porque la pulsión está siempre vinculada en él a la conducta y objetos que biológicamente le corresponden ("a priori”, por así decir). El hombre en cambio, aquejado por una reducción del instinto o pérdida completa de los instintos [Instinktlosigkeit], debe aprender con esfuerzo a dirigir la pulsión biológica hacia la conducta y objetos que biológicamente se adecuan a ella, ya que en él se da un hiato entre la pulsión y la acción consumatoria orientada a suprimir la pulsión, debido a su "apertura al mundo" (su propio mundo interior en este caso, que le permite el conocimiento objetivo de su vida pulsional y con él su distanciamiento del automatismo de la acción $)^{37}$. Ese aprendizaje requiere un esfuerzo, un autodominio, como hemos dicho, el autodominio que las morales tradicionales denominaban con el término "ascesis"; ascesis que se vería facilitada una vez más "desde fuera" por el apoyo que proporcionan las conductas imperadas por el entorno institucional. Las conductas reguladas institucionalmente facilitan la ascesis individual en cuanto conductas socialmente exigidas y sancionadas. Precisamente la ascesis (a diferencia de la libertad, la igualdad, el progreso o el "humanitarismo"), habría sido uno de los elementos constitutivos de la moral cristiana no asimilado por la tabla de valores de la modernidad, a pesar de ser un factor clave del equilibrio psíquico e incluso de la "humanización" 38 .

Podría decirse por tanto que, desde el punto de vista de la economía pulsional, las instituciones desempeñan el papel principal de fijar, estabilizar y canalizar ("sublimar", en definitiva, en terminología freudiana) las pulsiones biológicas privadas de una pauta instintiva de expresión, al modelar un comportamiento estable que "se encuentra en el hombre en el lugar en el que se encuentra la reacción instintiva en el animal” 39 .

37. Cf. Gehlen, 1956: 54 ss. y toda la tercera parte de la obra ("Leyes pulsionales. Carácter. El problema del espíritu”, Gehlen, 1956: 386 ss.).

38. "Si todavía se pudiera imaginar una salida [al hombre demasiado "natural" y “autocomplaciente" de las sociedades occidentales], la única sería el ascetismo, [...] forma de disciplina y estímulo, como concentración de autocontroles espirituales y volitivos" (Gehlen, A. [1952], 1983: 84-85). El ascetismo es una de las "categorías más elevadas" de la antropología, "una continuación del proceso de hominización” (Gehlen, A. [1952], 1983: 85).

39. Gehlen, 1956: 26; "El papel de todos los productos fabricados por el hombre desde los aparatos y símbolos hasta las formas sociales- en relación con su constitución es el siguiente: que permiten secundariamente el automatismo del comportamiento negado 
El matrimonio sería un ejemplo especialmente relevante del papel regulador que las instituciones desempeñan en relación con la vida pulsional, y en concreto con la pulsión sexual.

Es sabido que todas las culturas distinguen claramente entre relaciones sexuales libres y relaciones matrimoniales reconocidas y con efectos legales (incluso cuando sólo disponen de un derecho consuetudinario), y no por casualidad. El nacimiento y cuidado de los hijos, fin biológico de la relación sexual, requiere, mucho más en el hombre que en cualquier otro animal, de un prolongado periodo de crianza, que sólo es óptima de acuerdo con Gehlen cuando padre y madre cooperan en ella, y por tanto permanecen unidos. La estabilidad necesaria de esa unión, que garantiza adecuadamente el fin de la reproducción y educación de la prole, se logra en virtud de la institucionalización de la relación gracias a las reglas matrimoniales, reglas que estipulan derechos y deberes reconocidos públicamente entre los cónyuges y respecto de los hijos; y que sostienen un comportamiento regulado que fortalece la permanencia de la unión más allá de las veleidades de la inestable vida emocional o pulsional de los cónyuges, de modo que la relación se "objetivaría" a través de su "enajenación” en el marco institucional ${ }^{40}$.

En realidad, si la libertad, de acuerdo con un viejo motto de la filosofía moral, consiste en el sometimiento de la voluntad a las obligaciones que impone el deber frente a la arbitrariedad del deseo, el así el "enriquecimiento de la motivación" que supone la acción libre (liberación de pulsiones biológicas y sometimiento a deberes), sería facilitado por las conductas habitualizadas que fomentan las instituciones. La libertad presupone de algún modo la "descarga por estabilidad" 41 que ellas facilitan.

Pero la productividad de las instituciones para la vida humana va mucho más allá de los rendimientos materiales, psicológicos o morales que acabamos de considerar. Los mecanismos institucionales muestran también según Gehlen su eficacia insustituible en el desarrollo de las facultades cognitivas, y del lenguaje muy en particular.

primariamente y, con ello, sobre todo, la regularidad no precisamente obvia del comportamiento en relación con las cosas y de unos hombres con otros" (Gehlen, 1956: 47).

40. "Lo biológico, lo económico, la progenie, la alimentación y la necesidad son más fuertes [que el deseo o los sentimientos], y la relación tiene que objetivarse, cosificarse más allá de la exclusividad de tales individuos; en una palabra, enajenarse en una institución" (Gehlen, 1953, en Apel, 1985: 199-200, cursivas en el original).

41. Gehlen, 1956: 32. 
El lenguaje exhibe efectivamente propiedades "institucionales". En un pasaje que a nuestro modo de ver contiene in nuce la intuición fundamental sobre la que se elevan las "éticas discursivas" de Apel y Habermas, Gehlen atribuye al lenguaje las siguientes tres propiedades institucionales: el lenguaje “estabiliza” en primer lugar la pluralidad caótica de los datos sensibles para unificarlos en objetos ${ }^{42}$, pero tiene al mismo tiempo la virtud más genuinamente institucional de fungir como la herramienta principal de la comunicación; comunicación que permite la reflexión y discusión sobre realidades no físicamente presentes, pero que lo están virtualmente gracias al lenguaje. Y posibilita por último, en tercer lugar, la acción humana concertada a partir de un previo "estar ya de acuerdo" sobre el significado de las palabras. Nos permitimos citar el pasaje en cuestión en toda su extensión por su carácter que se nos antoja verdaderamente precursor:

Se debe entender en este sentido incluso el lenguaje como institución, porque establece núcleos de estabilización en la percepción, y más allá de ellos en el comportamiento recíproco de los hombres. El mero nombre que una cosa porta, la convierte, como a la percepción, en objeto en idéntico sentido, pero además proporciona la oportunidad de un comportamiento consensuado incluso en ausencia de esa percepción. Una cosa aparece en su nombre cuando puede ponerse en funcionamiento con el propio sonido sobre la misma cosa un acuerdo virtual con otros, y sobre todo una cooperación virtual con otros, ya se encuentre o no la cosa ante los ojos. Más aún, parece que en los estratos más profundos de las lenguas el nombre hubiese "fijado" la esencia, la hubiese exorcizado. (Gehlen, 1956: 195-6, cursivas nuestras)

Por lo demás, no sólo el lenguaje, base del acuerdo, de la acción coordinada y por tanto de las instituciones y de la cultura en general, es una realidad institucional, sino que las instituciones son igualmente necesarias para el progreso científico y artístico, puesto que el progreso en cualquier ámbito presupone el dominio automatizado de una infraestructura de capacidades jerárquicamente ordenadas que permiten, una vez convertidas en hábitos, la aparición y resolución de nuevos desafíos intelectuales o técnicos que no se podrían plantear, ni mucho menos resolver, sin el dominio de esas com-

42. El papel lenguaje (o del lenguaje-concepto), como configurador de "realidades" a partir de los estímulos captados sensiblemente también había sido tematizado ampliamente por Herder en su Ensayo sobre el origen del lenguaje. 
petencias previas, dominio que "descarga" la atención para dirigirla hacia problemas cada vez más sofisticados ${ }^{43}$.

El desarrollo de las competencias y capacidades teóricas o técnicas necesita empero él mismo, una vez más, de un entorno institucional en el que son conservadas y “educadas” y sin el que no podrían perfeccionarse (Gehlen, 1956: 282$)^{44}$.

\section{LA AMENAZA DE LA SUbJETIVIDAD EXACERBADA}

A la estabilidad de cualquier conducta lograda gracias a las instituciones opone enérgicamente Gehlen las tendencias dominantes de la cultura contemporánea, aquejada de una subjetividad exacerbada que tiende a producir sujetos anómicos privados de referencias institucionales; y destinados a convertirse en su única y solipsista autorreferencia de conducta. La cultura europea moderna, especialmente a partir de la Ilustración, habría potenciado hasta tal punto la esfera de la subjetividad, que cargaría a la persona con la obligación demasiado pesada de tener que elegir por sí misma siempre y en cada caso la acción adecuada a cada situación.

El proceso de subjetivización de la experiencia en occidente habría conocido tres hitos principales según Gehlen: la aparición del cristianismo con su descubrimiento del "hombre interior" y su desdivinización de las instituciones (privadas así de sus antiguas teogonías legitimadoras); la Ilustración y su promoción exacerbada de la conciencia de los derechos individuales (en detrimento de la conciencia de la pertenencia a todos sociales respecto a

43. "Costumbres especializadas desarrollan un umbral de estimulación creciente, tanto reacciones motóricas y sensibles finas como una escala diferenciada de esquemas de pensamiento" (Gehlen, 1956: 79); "las funciones espirituales superiores pueden sobrevolar y dejar tras de si el "aparato" del lenguaje, pero sólo pueden haber ganado a través de él la libertad de su amplitud de extensión” (loc. cit., idea que Gehlen toma en préstamo de la recensión de N. Hartmann sobre El hombre publicada en 1941 en las Blätter für deutsche Philosophie, Gehlen, 1956: 79).

44. Llamativamente Popper, en otro contexto, y desde presupuestos completamente distintos a los de Gehlen, apunta igualmente hacia el carácter institucional de la praxis científica como garantía de su objetividad: "Es interesante que lo que normalmente se llama objetividad cientifica se basa, hasta cierto punto, en instituciones sociales. ... es el carácter público de la ciencia y de sus instituciones el que impone una disciplina mental sobre el hombre de ciencia individual y el que salvaguarda la objetividad de la ciencia y su tradición de discutir críticamente las nuevas ideas” (Popper, 1973: 170-1). 
los cuales existen también deberes); y el trabajo tecnificado, que conlleva un extrañamiento del mundo natural. El conjunto de estos acontecimientos ha conducido al hombre de las sociedades avanzadas a una "pérdida de realidad" [Realitätsverlust], a un "extrañamiento del mundo" [Weltfremdheit] o pérdida del mundo [Weltverlust $]^{45}$.

La inflación de la subjetividad tiene que ver con un "estado de toma de conciencia crónica del yo" que iría acompañado de un "hartazgo del espacio de la representación" y un correlativo "empobrecimiento del círculo de acción”" El "empobrecimiento del círculo de la acción" es consecuencia, por otra parte, de una sociedad burocratizada y de un trabajo tan tecnificado y especializado que priva al trabajador, a diferencia de lo que sucedía con el artesano del Antiguo Régimen, de un contacto directo con la naturaleza y con la totalidad del proceso de manufacturación del producto, limitando su participación a la ejecución de simples actos maquinales repetitivos. Todo ello genera una "experiencia de segunda mano" con cierto grado de irrealidad frente a la experiencia "de primera mano" de que disfrutaba el trabajador de la época preindustrial. La sobreabundancia de información no asimilable (recordemos que Gehlen escribe esto ¡en 1957!), divulgada por los medios de comunicación de masas, se encarga de completar la tarea ${ }^{47}$. El abigarrado, sobreabundante y caótico universo de vivencias psíquicas reflejas de todo tipo acaba convirtiéndose con el tiempo en un mal "sustitutivo de la vida" [Lebensersatz] y de la acción. Si a ello se añaden los "síntomas de decadencia” que Gehlen percibe en la sociedad occidental motivados por el "luis-felipismo" ambiental en el que "el placer y la ganancia se han convertido en una exigencia jurídica” (Gehlen, 1956: 119), se comprende la crítica cultural conservadora que Gehlen lanzará contra todo el conjunto y que formulará solemnemente en 1952 en su conocida y airada declaración "institucionalista antiilustrada" (Habermas), en la que, de la mano de una manifiesta hostilidad contra la clase de los intelectuales, reivindica una "vuelta a la cultura" como remedio para los males de una sociedad sobrecargada de subjetivismo:

45. Gehlen, 1956: 126, esta constatación constituye la hipótesis histórica de partida de Moral e hipermoral.

46. Gehlen, 1956: 125, 127.

47. El flujo inasimilable de información genera un "mar de inseguridad” (Gehlen, 2004:54) gestionado por la "máquina de pensar de la prensa, las radios, los cines" (Gehlen, 1956: 49). 
Sustento una opinión decididamente contraria a la del siglo XVIII: es hora de un anti-Rousseau, de una filosofía pesimista que tome la vida en serio. Para Rousseau, la "vuelta a la naturaleza" significa: "la cultura pervierte al hombre [...]". Muy por el contrario, hoy nos parece a nosotros que en el caso del ser humano el estado natural es el caos [...]. Cultura es lo improbable, o sea, el derecho, la moralidad, la disciplina, la hegemonía de la moral. [...] Cuando se imponen los charlatanes, los diletantes, los intelectuales insustanciales; cuando empieza a sonar el viento de la frivolidad generalizada, entonces también se relajan las instituciones más antiguas y las corporaciones profesionales severas: la legislación se torna flexible; el arte, neurótico; la religión, sentimental. [...] ¡Volvamos a la cultura! (Gehlen, [1952]. 1993: 78) ${ }^{48}$

\section{Conclusión: actualidad de la teoría de las instituciones de GeHlen}

Como señalamos al comienzo, la propuesta antropológica de Gehlen es, entre los fundadores de la antropología filosófica, la que conoció una presencia más dilatada en la filosofía alemana hasta los años setenta e incluso hasta finales del siglo XX.

Nociones capitales de su antropología constituyen además motivos recurrentes, y en cierto modo tesis de fondo, del pensamiento de dos miembros destacados de la llamada "generación escéptica" de la posguerra como Odo Marquard y Hans Blumenberg (Marquard, 2001, Blumenberg, 2011).

$\mathrm{Su}$ institucionalismo radical unido a su ultraconservadurismo político suscitó las suspicacias inevitables de los autores en el entorno de la Escuela de Frankfurt, autores que no dejarán empero de reconocer la valía de muchos de sus análisis (Habermas, 1977: 100-109) ${ }^{49}$.

Gehlen fue sin duda un "pionero de la investigación sobre las instituciones” (Arlt, 2001: 153), y un agudo crítico cultural (conservador) de la sociedad de su tiempo, que en muchos de sus rasgos todavía es la nuestra. Se le ha achacado su insensibilidad respecto a la complejidad de los procesos sociales e históricos únicos que moldean en cada caso el surgimiento y permanencia de cada institución, el haber sido "ciego para los procesos sociales de formación [de instituciones]” (Rehberg, 1994: 541, en Arlt: 154)' si bien podría alegarse en su favor que en ningún momento pretende Gehlen haber

48. Trece años más tarde insistirá en el mismo diagnóstico en Moral e hipermoral en unos tonos si cabe más sombríos (Gehlen, 1973: 95 ss., en el cap. 7, "Instituciones”).

49. Cf. además Habermas, 2019. Habermas entró en contacto directo con la antropología filosófica a través del director de su tesis doctoral en Bonn, Erich Rothacker. 
elaborado una "sociología”, sino una "filosofía” de las instituciones, puesto que se mueve decididamente en el nivel epistémico de una antropología genuinamente filosófica, a pesar de todas su protestas de filósofo de tendencia "empírica”.

Su insistencia quizás demasiado unilateral en el poder conformador de las instituciones, tanto de la conducta individual como de los procesos de interacción social, hasta lo que podría parecer una justificación de la eliminación de cualquier autonomía o libertad individual, no podía sino evocar la imagen todavía viva de masas compactas de escuadras uniformadas bajo el signo de la esvástica.

Más de medio siglo después, cabría decir que muchos fenómenos sociales contemporáneos más o menos disfuncionales que afectan a sectores muy vulnerables de la población, parecerían, en muy buena medida, haber venido a darle la razón. Cuando psicólogos y pedagogos, por ejemplo, se quejan de la "falta de autocontrol" de niños y adolescentes, que se traduce en problemas de comportamiento y bajo rendimiento académico, y que es debida en buena medida a la dejación por parte de los padres de su función de supervisión y monitorización de la conducta de sus hijos (padres que no se atreven a "poner límites”, como suele decirse), ¿ no están pensando implícitamente, y casi a la letra, con las categorías de Gehlen de "estabilización de la conducta desde fuera"? 50

Cuando los psicólogos insisten en la importancia de "crear rutinas" como una condición del bienestar psíquico (habitualizar conductas por tanto, y "descargar" a la subjetividad del esfuerzo de descubrir por sí misma la acción correcta en cada situación), ¿no hablan de nuevo el lenguaje de Gehlen?

La "terapia ocupacional" y la "distracción conductual" como remedios contra determinados desequilibrios psíquicos ¿no tienen que ver con la liberación mediante la "acción" de una "subjetividad sobrecargada" y potencialmente enfermiza?

Para terminar, el llamado "capitalismo emocional" y todo el psicoturn que ha invadido la pedagogía en los últimos años ¿no explota y exacerba precisamente el sobreexceso de subjetividad que aqueja al sujeto de la cultura consumista de masas, al tiempo que lo recluye en un análisis morboso de sí

50. Cf. Baumeister y Vohs, 2004 sobre el autocontrol desde diversos enfoques psicológicos. 
mismo que le dificulta una verdadera acción orientada a suprimir las causas objetivas de su malestar?51

Una sociedad aherrojada en los lazos de una férrea disciplina institucional anula sin duda la libertad del sujeto, pero un déficit institucional hace la libertad igualmente improbable por el extremo contrario, porque impide la estabilidad y fortaleza psíquicas necesarias que son condición de posibilidad de la libertad.

Sorprendentemente, muchos años antes que Gehlen, un autor nada sospechoso de tendencias autoritarias como Durkheim, ya había apuntado en La división del trabajo social (1893) y El suicidio (1897), la relación inversa entre integración social y malestar psíquico (tendencia al suicidio significativamente) por falta de regulación o anomia, en términos que se dirían escritos por el propio Gehlen:

La anomía, como estado social, es una falta de dirección que suele aparecer en las épocas de revolución social. En el individuo se corresponde con un desconcierto e inseguridad o lo que hoy se suele definir como alienación o pérdida de sentido. La anomia es un estado de la sociedad donde los valores tradicionales han dejado de tener autoridad, mientras que los nuevos ideales, objetivos y normas carecen de fuerza (Durkheim, 2008, en Palacio, 2010: 5) 52

Si "una sociedad democrática, [...] una sociedad de libertades es incompatible con instituciones autoritarias fuertemente jerarquizadas, que favorecen a unos individuos y penalizan a otros" (Álvarez-Uría y Varela, 2009: 220), parece claro que es igualmente imposible una sociedad democrática compuesta de individuos frágiles psicológicamente, por falta de una adecuada

51. Para una crítica al giro emocional en la educación desde una perspectiva aristotélica y marxista respectivamente, $c f$. Carr, 2007, y Ecclestone y Hayes, 2009.

52. Durkheim observó con preocupación el sesgo individualista de la cultura industrial como consecuencia de la ruptura de los marcos morales de la sociedad preindustrial, especialmente el familiar. Los pasajes gehleanos avant la léttre en su obra son sobreabundantes: "la libertad (nos referimos a la libertad justa, a la que tiene la sociedad el deber de hacer respetar) es ella misma el producto de una reglamentación” (Durkheim, 1985, vol. 1: 4). "Una regla, en efecto, no es sólo una manera de obrar individual; es, ante todo, una manera de obrar obligatoria, es decir, sustraída, en cierta medida al libre arbitrio individual" (Durkheim, 1985, vol. 1: 5, cursivas en el original); "si la anomía es un mal, lo es, ante todo, porque la sociedad la sufre, no pudiendo prescindir, para vivir, de cohesión y regularidad" (Durkheim, 1985, vol. 1: 6), los diagnósticos de la sociología de Bauman sobre las patologías de las sociedades occidentales contemporáneas apuntan exactamente en la misma dirección (Bauman, 2006). 
socialización en instituciones (familia, sistema educativo), que renuncian a su función de orientar, exigir y poner los límites necesarios que hacen posible un individuo psicológicamente fuerte, y autónomo en consecuencia, protegido frente a conductas manipuladoras o populismos políticos de cualquier signo. Se trata de encontrar el difícil equilibro posible (aunque necesario) entre la "descarga" liberadora de la conducta que permiten las instituciones, y la posibilidad de una crítica racional meliorativa de las mismas (Apel, 1991:149).

\section{REFERENCIAS BIBLIOGRÁFICAS}

Álvarez MunÁrriz, L. (1996). "Actualidad de la antropología filosófica de A. Gehlen”. Thémata: Revista de Filosofía, 17, pp. 289-304.

Álvarez Uría, F. y Varela, J. (2009). Sociología de las instituciones. Bases sociales y culturales de la conducta. Madrid: Morata.

Apel, K. O. (1985). "La "filosofía de las instituciones" de Arnold Gehlen y la metainstitución del lenguaje". En Apel, K. O., La transformación de la filosofía. Madrid: Taurus, vol. 1, pp. 191-214.

Apel, K. O. (1991) "La ética del discurso como ética de la responsabilidad. Una transformación postmetafísica de la ética de Kant”. En K. O. Apel, Teoría de la verdad y ética del discurso. Barcelona: Península, pp. 147-184.

Artt, G. (2001). Philosophische Anthropologie. Stuttgart-Weimar: J.B. Metzler.

Barnes, E. H. (1942). Social Institutions. Englewood Cliffs; Prentice Hall.

Bauman, Z. (2006). Vida líquida. Barcelona: Paidós.

BAUMeISTER, R. F. y Vohs, K. D. (eds.) (2004). Handbook of self-regulation: Research, Theory, and Applications. New York: Guilford.

Berger, P. L. y Kellner, H. (1965). "Arnold Gehlen and the theory of institutions". Social Research 32 (1), pp. 110-115.

Berger, P. L. y LucKMANn, Th. [1968] (1986). La construcción social de la realidad. Buenos Aires: Amorrortu.

Blumenberg, H. (2011). Descripción del ser humano. México: FCE.

BöHLER, D. (1981). “Arnold Gehlen: Handlung und Institution”. En SPeCK, J. (Hsg.). Grundprobleme der grossen Philosophen. Philosophie der Gegenwart II. Göttingen: Vandenhoeck and Ruprecht, pp. 231-282.

Carr, D., (2007). "Virtue, Emotions and Moral Ambivalence". Philosophy, 84, 1, pp. 31-46.

De Jouvenel, B. (1955). De la souverainité. Paris: Génin. 
De Maistre, J. (1814). Essai sur les principes générateurs des constitutions politiques et des autres institutions humaines. Paris: A la Sociéte Typographique (disponible en https://gallica.bnf.fr/ark:/12148/bpt6k41852g/f15.item.texteImage).

Diderot, D. [1771] Supplément au voyage de Bougainville (disponible en https:// www.bacdefrancais.net/diderot-supplement-voyage-bougainville.pdf).

Durkheim, É. [1902] (1985). La división del trabajo social. Madrid: Planeta-De Agostini, 2 vols.

DurkHEIM, É. (2008). El suicidio. Madrid: Akal.

Ecclestone, K. and Hayes, D. (2009). "Changing the subject: the educational implications of developing emotional wellbeing”. Oxford Review of Education, 35 (3), pp. 371-89.

Fischer, J. (2006). “Philosophische Anthropologie. Ein wirkungsvoller Denkansatz in der deutschen Soziologie nach 1945”. Zeitschrift für Soziologie, 35, pp. 322-347

Fischer, J. (2009). Philosophische Anthropologie. Eine Denkrichtung des 20. Jahrbunderts. Freiburg/München: Verlag Karl Alber.

Fischer, J. (2016). Exzentrische Positionalität. Studien zu Helmuth Plessner. Weilerswist: Velbrück Wissenschaft.

Gehlen, A. (1953), “Über die Geburt der Freiheit aus der Entfremdung”. Archiv für Rechts-und Sozialphilosophie, XL, 3.

GeHlen, A. (1980a) El hombre. Su naturaleza y su lugar en el mundo. Salamanca: Sígueme.

GeHlen, A. [1935] (1980b). "Der Idealismus und die Lehre des menschlichen Handelns”. En Gehlen, A. Philosophische Schriften. Gesamtausgabe, vol. 2, Hrsg. von Lothar Samson, Frankfurt a. M.: Vittorio Klosterman, pp. 311-342.

GeHlen, A. (1956). Urmensch und Spätkultur. Philosophische Ergebnisse und Aussagen. Bonn: Athenäum-Verlag.

Gehlen, A. (1966). Der Mensch. Seine Natur und seine Stellung in der Welt. Frankfurt a. M.-Bonn: Athenäum Verlag.

GeHlen, A. [1969] (1973). Moral und Hypermoral. Eine pluralistische Etbik. Frankfurt a. M.: Athenäum Verlag.

GeHlen, A (1993). Antropología filosófica. Del encuentro y descubrimiento del hombre por sí mismo. Barcelona: Paidós.

GeHLEN, A. [1952] (1993) "La imagen del hombre a la luz de la antropología moderna”. En Gehlen, Antropología filosófica. Barcelona: Paidós, pp. 73-86.

GeHlen, A. [1957] (1993) "Contribución a la historia de la antropología”. En Gehlen, A., Antropología filosófica. Barcelona: Paidós, pp. 23-40.

GeHlen, A. (1994). Imágenes de época: sociología y estética de la pintura moderna. Barcelona: Península.

Gehlen, A. (2001). Morale e hipermorale. La'etica pluralistica. Verona: Ombre Corte. GeHlen, A. [1957] (2004). “Die Seele im technischen Zeitalter”. En Gehlen, A. Die Seele im technischen Zeitalter und andere soziologische Schriften und 
Kulturanalysen". Gesamtausgabe, vol. 6 (Hsg. K.S. Rehberg) Frankfurt a. M.: Vittorio Klostermann, pp. 1-137.

Gehlen, A. (2021). L’homme. Sa nature et sa position dans le monde. Paris: Gallimard.

Gehlen, A, y Adorno, Th. W. (1988). “¿Es la sociología una ciencia del hombre? Una conversación radiofónica entre Theodor W. Adorno y Arnold Gehlen”. En Harich, W., Crítica de la impaciencia revolucionaria. Barcelona: Crítica, pp. 175-194.

Gehlen A., Schelsky, H. y Jantke, C. (1962). Sociología moderna. Buenos Aires: Depalma.

GonZÁlÉz-JARA, A. (1970). "El hombre según la teoría antropobiológica de Arnold Gehlen”. Anuario Filosófico, 3, pp. 93-165.

Habermas, J. [1973] (1977) "Philosophische Anthropologie (ein Lexikon-Artikel)". En Habermas, J., Kultur und Kritik. Verstrente Aufsätze. Frankfurt a. M.: Suhrkamp, pp. 89-111.

HABERMAS, J. [1994] (1999) "El vínculo interno entre estado de derecho y democracia”. En Habermas, J., La inclusión del otro. Estudios de teoría política. Barcelona: Paidós, pp. 247-58.

Hauriou, M. (1925). La théorie de l'institution et la fondation. Paris: Bloud et Gay. Herder, J. G. [1772] (1964), “Abhandlung über den Ursprung der Sprache”. En Herder, J. G., Sprachphilosophie. Ausgewählte Schriften. Mit einer Einleitung, Anmerkungen und Registern versehen von Erich Heintel. Hamburg: Felix Meiner, pp. 3-87.

Lourau, R. [1970] (1988). El análisis institucional. Buenos Aires: Amorrortu.

Lомво, Luis Á. (2008). "El hombre entre la antropología y la técnica”. Anuario filosófico. 41(2), pp. 363-381.

Malinowski, B. [1944] (1949). Eine wissenschaftliche Theorie der Kultur und andere Aufsätze. Zürich. Pan-Verlag.

Marquard, O. (1965). "Zur Geschichte des Begriffs philosophische Anthropologie”, En E. W. Böckenförde et al., Collegium Philosophicum. Studien Joachim Ritter zum 60. Geburstag. Basel/Stuttgart: Schwabe \& Co. Verlag, pp. 209-239 (traducc. esp.: Marquard, O., 2007. Las dificultades con la filosofía de la historia. Valencia: Pre-textos, pp. 133-155).

MARQUARD, O. (2001). Filosofía de la compensación: escritos sobre antropología filosófica. Barcelona: Paidós.

Mead, G. H. [1934] (1967). Mind, Self and Society. Chicago and London: The University of Chicago Press.

Miranda Rebeco, P. (2012). "La descarga social de la subjetividad y sus figuras". Alternativas cubanas en Psicología, 3,9, pp. 60-72,

Miranda Rebeco, P. (2012). La pre-comprensión de lo humano en la sociología de Lubmann. Raíces antropológicas del antibumanismo téorico lubmannniano. Santiago: Universidad Alberto Hurtado. 
Palacio, A. F. (2010). “La comprensión clásica del suicidio. De Émile Durkheim a nuestros días”. Affectio Societatis, vol. VII/12, pp. 1-12.

Pansera, M. T. (1991). L'uomo progetto della natura. L'antropologia filosofica di Arnold Geblen. Roma: Studium.

Panser, M.T. (2001). Antropologia filosofica. La peculiarità dell'nomo in Scheler, Geblen e Plessner. Milán: B. Mondadori.

PARsons, T. [1951] (1989). El sistema social. Madrid: Alianza Editorial.

Plessner, H. (2017). Les dégres de l'organique et l'Homme. Introduction a l'anthropologie philosophique. Paris: Gallimard.

Popper, K. R. [1957] (1973). La miseria del historicismo. Madrid: Alianza Editorial.

RAulet, Gérard (2009). La filosofía alemana después de 1945. Valencia: Universitat de València.

Rehberg, K.-S. (1993), “Nachwort des Herausgebers”. En Gehlen, A. Der Mensch. Seine Natur und seine Stellung in der Welt. Gesamtausgabe, vol 3-2, Frankfurt a. M.: Vittorio Klosterman, pp. 751-786.

RehberG, K.-S. (1994). “Diskussionsbeitrag”, en Helmut Klages/Helmut Quaritsch (Hg.) Zur geisteswissenschaftlichen Bedeutung Arnold Geblens. Vorträge und Disckussionsbeiträge des Sonderseminars 1989 der Hochschule für Verwaltungswissenschaften Speyer. Berlin: Duncker \& Humblot, pp. 517-541.

Renard. G. (1930). La théorie de la institution. Paris: Sirey.

Rodríguez Molinero, J. L. (1989). “La antropología de la acción de Arnold Gehlen”. Naturaleza y gracia, 36/3, pp. 423-469.

Roggero, E. (1986). “Institución”. En Franco Demarchi y Aldo Ellena (eds.). Diccionario de Sociología (adaptación española de Juan González Anleo). Madrid: Paulinas, pp. 922-8.

Rosales Rodríguez, A. (1999). “Técnica y naturaleza humana según Arnold Gehlen”. Folios. Revista de la Facultad de Arte y Humanidades, 11. https://doi. org/10.17227/01234870.

SÁnCHEZ CAPDEQUí, C. (1997a). "Sociología de las instituciones en la obra de Arnold Gehlen”. Inguruak, 17, 9, pp. 9-24.

Sánchez Capdequí, C. (1997b). "Procesos de institucionalización simbólica de la sociedad: acercamiento a la obra de Arnold Gehlen”, Bitarte: Revista cuatrimestral de Humanidades, 12, pp. 49-70.

Schnädelbach, H. [1983] (1991). Filosofía en Alemania 1831-1933. Madrid: Cátedra. SEIDEL, A. (1927). Bewusstsein als Verhängnis, aus dem Nachlass v. H. Prinzhorn. Bonn.

Vox (1990). Diccionario ilustrado latino-español, español-latino, Prólogo de Vicente García de Diego, Barcelona: Bibliograf.

Wiki Deutsch (2020). Helmuth Schelsky. 